\title{
The future of venoms-based drug discovery: an interview with Glenn King
}

Interview by Hannah Coaker (Commissioning Editor, Future Science Group).

Glenn King secured a PhD from the University of Sydney before doing his postdoctoral studies at the University of Oxford. After academic stints at the University of Sydney and the University of Connecticut Health Center, he joined the Institute for Molecular Bioscience at The University of Queensland in 2007, where he is currently an NHMRC Principal Research Fellow. The current focus of King's research is venom-based drug discovery, in particular the development of analgesics and anti-stroke therapies. Several molecules discovered in his lab have already progressed to preclinical studies. King currently serves on the Scientific Advisory Board of three companies and the Editorial Board of four journals. He is a former President of the Australian Society for Biophysics, current President of the Australian \& New Zealand Society for Magnetic Resonance and a member of the Executive Council of the International Society for Toxinology. In addition, King is a Fellow of the American Academy of Microbiology, and in 2013, he received the Sir Bob Robertson Award from the Australian Society for Biophysics and the Beckman Coulter Discovery Science Award from the Australian Society for Biochemistry \& Molecular Biology.

Q How did you become involved in the field of animal venom-based drug discovery?

I became interested in spider venoms as a potential source of natural insecticides in the middle of the 1990s. That work proved to be very successful. In 2005, while working at the University of Connecticut, I founded a spin out company called Vestaron, which recently received approval from the United States EPA (see [1]) for its first venom-peptide-based insecticide. Our initial decade of work on spider venoms revealed that they are rich in hyperstable, disulfide-rich peptides that potently and specifically modulate the activity of neuronal ion channels. Since many of these ion channels are therapeutic targets, it was a natural progression when I moved to the medically oriented Institute for Molecular Bioscience in 2007 to examine venoms as a potential source of therapeutic leads.
Q What properties make animal venoms a rich resource for novel lead compounds that may be used in drug design and development?

Most venomous animals rely heavily on their venom for predation, which applies a constant evolutionary selection pressure on toxin efficacy. As a result, toxins from spiders, centipedes and scorpions typically have extremely high specificity and potency for their molecular target due to hundreds of millions of years of evolutionary fine tuning. Moreover, venom peptides are typically rich in disulfide bonds, which provide them with a much higher level of biological stability than most peptides. Thus, to summarize, venom peptides are good drug leads because of their stability, potency and selectivity.

Can you describe your research group's current work? Is there an area of

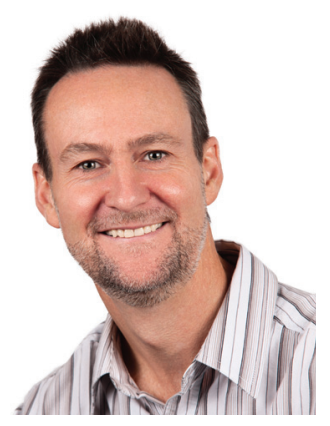

Glenn F King

Division of Chemical \& Structural Biology, Institute for Molecular Bioscience, Brisbane, Queensland, Australia. glenn.king@imb.uq.edu.au 
the field that you are particularly excited about at the moment?

We are interested in venom peptides that inhibit the analgesic target $\mathrm{Na}_{\mathrm{v}} 1.7$ and venom peptides that inhibit acid sensing ion channels (targets for both pain and stroke). We also remain interested in venom peptides with potential application as bioinsecticides.

I am excited about the whole field of venom-based drug discovery as new high-throughput screening techniques combined with advances in proteomics and transcriptomics will greatly accelerate the rate at which useful venom-derived drug leads are discovered. I am very excited about new toxin scaffolds and pharmacological activities that we have discovered in the venom of centipedes (in collaboration with Bryan Fry at The University of Queensland [Australia] and Lai Ren at the Chinese Academy of Sciences [China]) and ants (in collaboration with Pierre Escoubas at VenomeTech [France]). A specific development I am particularly enthused about is the recent successful Phase Ia clinical trial of a sea anemone peptide (ShK) for treatment of multiple sclerosis and other autoimmune diseases.

Q In what way have advances in proteomics accelerated the field of venom-based drug discovery?

Most venomous animals are very small (e.g., centipedes, spiders, scorpions and ants) and consequently they yield very tiny amounts of venom. Thus, they are not easily studied using traditional bioassay-guided venom fractionation techniques. Advances in proteomics and transcriptomics have massively reduced the sample requirements for studying venoms and consequently many previously unstudied venoms, including previously unstudied taxa, have now become accessible.

Q Part of your research looks at the analgesic qualities of centipede and spider venoms. Can you describe their mode of action and explain the therapeutic potential of these venoms?

We have been screening both centipede and spider venoms for selective blockers of the analgesic target $\mathrm{Na}_{\mathrm{v}}$ 1.7. Humans born without functional $\mathrm{Na}_{\mathrm{v}} 1.7$ channels have a congenital insensitivity to pain, without any other sensory deficits except impaired smell, and consequently this channel appears to be an ideal analgesic target. Because this channel works at a very high level in the pain signaling circuit, blockers of this channel should be useful for management of difficultto-treat types of pain, such as neuropathic pain. The spider and centipede venom peptides work by binding to the voltage-sensor regions of the channel, making it insensitive to changes in membrane potential; in lay terms, the channels cannot turn on because these toxins hold the voltage switch in the 'off' position.

Q The taxonomical range of venomous animals studied is relatively narrow. Do you think that this hinders venom-based drug discovery or is there significant convergence among the structure of animal toxins?

The taxonomical range of venomous animal that has been studied is rapidly expanding due to advances in proteomics and transcriptomes. While there has been very significant convergence of certain toxin types (reviewed in [2]), there will undoubtedly be new toxin scaffolds discovered as each new taxon is studied. For example, recent work on ants and centipedes has revealed entirely new classes of venom peptides.

\section{Q Do you feel that sufficient work is being} conducted by academia and industry toward fully exploiting the therapeutic potential of animal venoms?

This is a difficult question and the answer is not simple. There is significant investment worldwide in toxinology research in academia and there continues to be increasing focus on the potential therapeutic application of venom-derived molecules. However, there is a general lack of concerted international collaboration in this area, something that the International Society for Toxinology is seeking to address. Bringing together researchers with expertise in venoms-based drug discovery with those skilled in peptide-drug development, for example, would be highly synergistic.

From an industry perspective, there are actually quite a few companies that are focused on therapeutics derived from venoms and several big pharma companies are also interested in this area. In spite of this, the amount of money invested by big pharma in this area is miniscule. Venom-based drug discovery is a relatively new field and sometimes a lack of experience in drug development leads toxinology researchers to make unsubstantiated claims about the therapeutic potential of venom molecules, which encourages skepticism in big pharma. As the field matures and better preclinical data become available, big pharma is likely to invest more in this area.

\section{Q How do you anticipate the field of} venom-based drug discovery will progress in the next $5-10$ years?

There will be an exponential increase in the number of venom peptide sequences available over the next 5-10 years due to advances in proteomics, transcriptomics and increasingly genomics. In combination with advances in high-throughput screening of venoms/ venom peptides, this will lead to a much larger pipe- 
line of venom-derived drug leads. The challenge, as in any drug discovery project, will be to choose the best leads for subsequent optimization and development.

Q What words of advice do you have for a young scientist envisioning a career in venom-based drug discovery?

Follow your passion, as this drives good science. But choose a project that is either fundamentally important and will lead to new understanding in a particular field (e.g., how did venom evolve in an unstudied taxon) or a translational project that has the potential to be transformative (e.g., first-in-field drugs for a human disease).

\section{References}

1 Vestaron Gains U.S. EPA Approval of Naturally Occurring Insecticide. (2014).

www.vestaron.com/epa-approval/

\section{Disclaimer}

The opinions expressed in this interview are those of the interviewee and do not necessarily reflect the views of Future Science Ltd.

\section{Financial \& competing interests disclosure}

The author has no relevant affiliations or financial involvement with any organization or entity with a financial interest in or financial conflict with the subject matter or materials discussed in the manuscript. This includes employment, consultancies, honoraria, stock ownership or options, expert testimony, grants or patents received or pending or royalties.

No writing assistance was utilized in the production of this manuscript.

2 Fry BG, Roelants K, Champagne DE, et al. The toxicogenomic multiverse: convergent recruitment of proteins into animal venoms. Annu. Rev. Genomics Hum. Genet. 10,483-511 (2009). 